DOI: https://doi.org/10.14311/TPFM.2022.016

\title{
SHIFTED PROPER ORTHOGONAL DECOMPOSITION AND ARTIFICIAL NEURAL NETWORKS FOR TIME-CONTINUOUS REDUCED ORDER MODELS OF TRANSPORT-DOMINATED SYSTEMS
}

\author{
Anna Kovárnová ${ }^{1}$, Philipp Krah ${ }^{2}$, Julius Reiss ${ }^{3}$, Martin Isoz ${ }^{1,4}$ \\ ${ }^{1}$ Department of Mathematics, Faculty of Chemical Engineering, University of Chemistry and \\ Technology, Technická 5, Prague 166 28, Czech Republic \\ 2 Institut für Mathematik, Faculty II of Mathematics and Natural Sciences, Technische Uni- \\ versität Berlin, Strasse des 17. Juni 136, 10623 Berlin, Germany \\ 3 Institut für Strömungsmechanik und Technische Akustik, Faculty V of Mechanical Engineer- \\ ing and Transport Systems, Technische Universität Berlin, Müller-Breslau-Strasse 15, 10623 \\ Berlin, Germany \\ ${ }^{4}$ Czech Academy of Sciences, Institute of Thermomechanics, Dolejškova 5, Prague 18200 , \\ Czech Republic
}

\begin{abstract}
Transport-dominated systems are pervasive in both industrial and scientific applications. However, they provide a challenge for common mode-based model order reduction (MOR) approaches, as they often require a large number of linear modes to obtain a sufficiently accurate reduced order model (ROM). In this work, we utilize the shifted proper orthogonal decomposition (sPOD), a methodology tailored for MOR of transport-dominated systems, and combine it with an interpolation based on artificial neural networks (ANN) to obtain a time-continuous ROM usable in engineering practice. The resulting MOR framework is purely data-driven, i.e., it does not require any information on the full order model (FOM) structure, which extends its applicability. On the other hand, compared to the standard projection-based approaches to MOR, the dimensionality reduction utilizing SPOD and ANN is significantly more computationally expensive since it requires a solution of high-dimensional optimization problems.
\end{abstract}

Keywords: model order reduction, shifted POD, CFD-DEM, OpenFOAM.

\section{Introduction}

The ongoing progress in numerical methods and available computing power allows for development of more and more complex numerical models, which tend to be expensive from the point of view of data storage and simulation time. However, the industrial practice often calls for system control or optimization based on these high-fidelity models. This situation promotes development of various approaches to model order reduction (MOR), i.e. to derivation of low-dimensional models from experimental or numerical data $[1,2]$.

In fluid dynamics, MOR approaches are often based on modal decomposition of available data, which allows to characterize the model internal dynamics and works for non-linear systems $[3$, $4,5]$. An example of such approach is the proper orthogonal decomposition (POD) combined with the Galerkin projection and discrete empirical interpolation method (DEIM) [3] as used for example in [1]. Nevertheless, most methods are based on an approximation of the snapshot matrix, $Y=\left(y_{i j}\right)=\left(y\left(x_{i}, t_{j}\right)\right), Y \in \mathbb{R}^{m \times n}$, comprising the complete system data by

$$
Y \approx Y^{\ell}=\sum_{r=1}^{\ell} \psi_{r} \otimes \eta_{r}=\Psi^{\ell} H^{\ell}, \quad \Psi^{\ell}=\left[\psi_{1}, \ldots, \psi_{\ell}\right] \in \mathbb{R}^{m \times \ell}, H^{\ell}=\left[\eta_{1}, \ldots, \eta_{n}\right] \in \mathbb{R}^{\ell \times n},
$$

i.e., by a sum of $\ell$ dyadic pairs $\left(\psi_{r} \otimes \eta_{r}\right)_{i j}=\psi_{r}\left(x_{i}\right) \eta_{r}\left(t_{j}\right)$, where $\left\{\psi_{r}\right\}_{r=1}^{\ell}$ are the spatial modes, toposes, and $\left\{\eta_{r}\right\}_{r=1}^{\ell}$ are the time-dependent amplitudes, chronoses. In other words, the columns of the matrix $Y$ are approximated via a superposition of $\ell \ll n$ toposes.

However, the dynamical behavior of transport-dominated systems usually cannot be described via a superposition of a few spatial modes. Consequently, the transport-dominated phenomena frequently pose a challenge for the standard mode-based methods $[2,4,6]$. Recently, several approaches to enable MOR of transport-dominated systems were proposed, see e.g. [2, 7, 8] and 
references therein. Usually, these approaches are based on introducing some time-dependent shift, or, transport operator, to compensate for the transport. One of these approaches is the shifted proper orthogonal decomposition (sPOD) introduced by Reiss et al. [2] and reformulated in a more rigorous manner in [4]. In sPOD, the time-dependent shift is combined with a procedure to separate the different transports in the system, i.e. to sort the input data into several co-moving frames depending on the definition of the transport operators.

The aim of the present work is to practically apply sPOD for MOR of transport-dominated systems. While the ultimate goal is to devise a framework applicable to time-parameter or multiparameter dependent partial differential equations (PDEs), in the present contribution we focus solely on the time-dependent systems. Consequently, the sought reduced order model (ROM) has to be computationally cheap and continuous in time. While sPOD exhibits great promise in approximation of data originating from transport-dominated systems via just a few spatial modes in the respective co-moving frames, it practically disallows for utilization of the standard projection-based approaches to the reduced order model construction [2]. In order to allow for practical application of SPOD in MOR, we propose to replace the projection framework by an interpolation between the discrete values $\left\{\eta_{r}\right\}_{r=1}^{\ell}$ utilizing an artificial neural network (ANN), an idea usable for parametrized PDEs and similar to the one presented in [9] and in [10].

In the following, we briefly present the model order reduction problem, outline the fundamentals of MOR based on POD combined with Galerkin projection, and give the basics of the shifted POD while emphasizing the challenge it poses for projection-based MOR. Afterwards, the proposed framework based on coupling POD and sPOD with ANN-based interpolation is described and illustrated on numerical examples motivated by model order reduction of CFD-DEM data [11, 12].

\section{Methods}

Problem formulation and specifics The general goal of the model order reduction is to reduce the computational complexity of a system (full order model, FOM) by reducing its degrees of freedom (DoFs). As an example, let us focus on a general non-linear partial differential equation (PDE) for an unknown function $y: I=(0, T] \subset \mathbb{R} \times \Omega \subset \mathbb{R}^{3} \rightarrow \mathbb{R}^{k}$,

$$
\begin{gathered}
\dot{y}+\mathcal{L}(t, y)=0, \quad \forall(t, \boldsymbol{x}) \in I \times \Omega \\
y(t, \boldsymbol{x})=g(t, \boldsymbol{x}), \forall(t, \boldsymbol{x}) \in I \times \partial \Omega, \quad y(\boldsymbol{x}, 0)=y_{0}, \forall \boldsymbol{x} \in \Omega
\end{gathered}
$$

where the operator $\mathcal{L}: \mathbb{R} \times \Omega \rightarrow \mathbb{R}^{k}$ represents all terms of the original PDE apart from the temporal derivative, $\Omega$ is an open bounded domain and $\partial \Omega$ its boundary, and $g: I \times \partial \Omega \rightarrow \mathbb{R}^{k}$ and $y_{0}: \Omega \rightarrow \mathbb{R}^{k}$ boundary and initial conditions, respectively. After a semi-discretization of $(2)$ via an arbitrary numerical method (FVM, FEM, FDM,...), we obtain the system

$$
\dot{y}^{h}+\mathcal{L}^{h}\left(t, y^{h}\right)=0, \quad \forall t \in I, y^{h}(0)=y_{0}^{h} .
$$

Now, let $\tilde{m}$ be the number of DoFs in $\Omega^{h}$, the spatial discretization of $\Omega$. Then $y^{h}: I \rightarrow \mathbb{R}^{m}$, $m=\tilde{m} k$, in (3) is the spatial semi-discretization of the function $y$ and $\mathcal{L}^{h}: I \times \Omega^{h} \rightarrow \mathbb{R}^{m}$ is the spatial discretization operator corresponding to $\mathcal{L}$. Hereafter, we will work solely in the spatially discrete setting and omit the superscript $h$.

Note that the system (3) can be usually expressed in the form

$$
\dot{y}=A y+b(t, y), \quad \forall t \in I, y(0)=y_{0},
$$

where $A y, A \in \mathbb{R}^{m \times m}$, represents the linear part of the system and $b(t, y)$ the system non-linearities. In the following, we will refer to the system (4) as to the full order model (FOM).

The examined approach to MOR is an a-posteriori one. Thus, to construct the ROM, it is necessary to have not only the FOM (4), but also its solution snapshots saved at given times, $\boldsymbol{S}:=\left\{y_{j}:=y\left(t_{j}\right)\right\}_{j=1}^{n}, t_{j} \in(0, T]$, where $n$ is the number of system snapshots. In fact, the general MOR goal, i.e. to approximate the set $\boldsymbol{S}$ as well as possible by the means of a low-dimensional surrogate $\eta^{\ell}: I \rightarrow \mathbb{R}^{\ell}, \ell \ll m$, can be achieved without the knowledge of (4), provided the dataset $\boldsymbol{S}$ is sufficiently large. 
Projection-based model order reduction and proper orthogonal decomposition In the standard projection-based MOR, see e.g. [13], the surrogate $\eta^{\ell}$ is sought as a solution of a modified equation

$$
\dot{\eta}^{\ell}=A^{\ell} \eta^{\ell}+f^{\ell}\left(t, \eta^{\ell}\right), \forall t \in(0, T], \quad \eta^{\ell}(0)=\eta_{0}^{\ell}
$$

constructed by a projection of the original system (4), the solution of which lies in the space $V, \operatorname{dim}(V)=m$, onto a low-dimensional subspace $V^{\ell} \subset V, \operatorname{dim}\left(V^{\ell}\right)=\ell \ll m$. The projection itself is usually linear (Galerkin), i.e. $A^{\ell}:=\left(\Psi^{\ell}\right)^{\mathrm{T}} A \Psi^{\ell} \in \mathbb{R}^{\ell \times \ell}$ and $f^{\ell}:=\left(\Psi^{\ell}\right)^{\mathrm{T}} b\left(t, \Psi^{\ell} \eta\right), \Psi^{\ell} \in \mathbb{R}^{m \times \ell}$. The unknown function $\eta^{\ell}: \mathbb{R} \rightarrow \mathbb{R}^{\ell}$ is formally defined as $\eta^{\ell}(t):=\left(\Psi^{\ell}\right)^{\mathrm{T}} y(t)$ and the initial condition is projected as $\eta^{\ell}(0)=\left(\Psi^{\ell}\right)^{\mathrm{T}} y_{0}$.

The quality of approximation of $y$ via $\eta^{\ell}$ and the reduced order model dimension is directly dependent on the choice of the projector $\Psi^{\ell}$. One of the most popular choices for the $\Psi^{\ell}$ construction based on the data $\boldsymbol{S}$ is the proper orthogonal decomposition $[13,14]$ which technically reduces to a singular value decomposition (SVD) of a matrix $Y=\Psi \Sigma X^{\mathrm{T}}$ constructed from $\boldsymbol{S}$ as $Y=\left[y_{1}, \ldots, y_{n}\right] \in \mathbb{R}^{m \times n}[2]$. After truncation, the SVD of $Y$ can be rewritten in the form used in equation (1), i.e. as

$$
Y \approx Y^{\ell}=\Psi^{\ell} H^{\ell}, \quad H^{\ell}=\Sigma^{\ell}\left(X^{\ell}\right)^{\mathrm{T}} \in \mathbb{R}^{\ell \times n}, \quad \Sigma^{\ell}=\operatorname{diag}\left(\sigma_{1}, \ldots, \sigma_{\ell}\right), X^{\ell} \in \mathbb{R}^{n \times \ell},
$$

where each column $\psi_{r}, r=1, \ldots, \ell$ of $\Psi^{\ell}$ corresponds to a single spatial mode, topos, of the modal decomposition of $Y$ and each row $\eta_{r}, r=1, \ldots, \ell$ of $H$ is a time-dependent amplitude, chronos. Note that only the first column of $H^{\ell}$ is used in POD-Galerkin ROM construction. However, the full matrix $H^{\ell}$ is available from SVD of $Y$.

Finally, inspecting the reduced non-linearity definition in (5), it may be noted that its evaluation is inefficient as it is necessary to first evaluate the full order non-linearity for the variable $\Psi^{\ell} \eta \in \mathbb{R}^{m}$ and then to project the result back to $V^{\ell}$. However, a number of approaches have been proposed to improve the efficiency of the reduced order model non-linearity evaluation, see e.g. the DEIM by Chaturantabut and Sorensen [3].

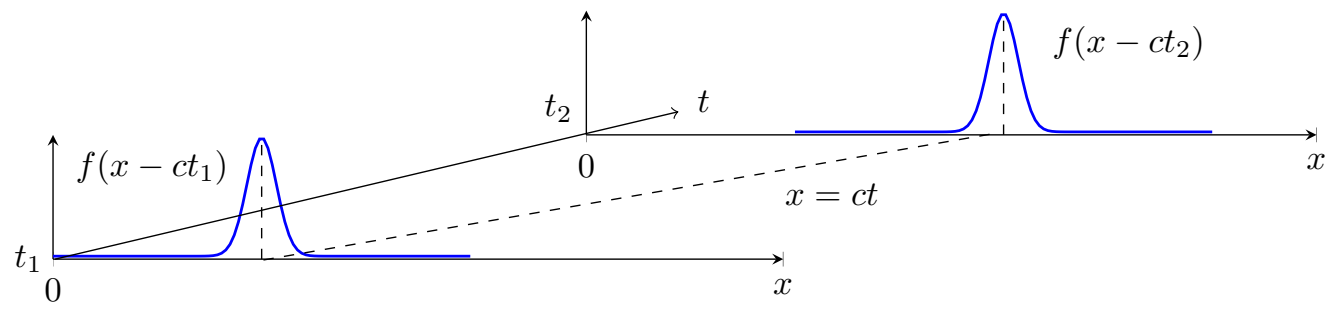

Figure 1: Advected sharp Gaussian pulse.

Shifted proper orthogonal decomposition To illustrate the shortcomings of the standard modal decomposition approaches such as POD, see (1), let us consider a one dimensional advection equation for a transported quantity $\varphi$,

$$
\partial_{t} \varphi+c \partial_{x} \varphi=0, \quad \forall(t, \boldsymbol{x}) \in I \times \Omega=(0, T] \times(0, L],
$$

where $L$ is the domain length and $c$ is the transport velocity. For arbitrary initial conditions $\varphi(0, x)=f(x)$, a general solution of (7) may be written in the form of a traveling wave $\varphi(t, x)=$ $f(x-c t)$. The situation for the case of $c>0$ and $f(x)=\exp \left(-\left(x-x_{0}\right)^{2} / \delta\right)$ being a Gaussian pulse is illustrated in Fig. 1.

Using the Gaussian pulse, fixing $\delta=L / 50$, generating a matrix of snapshots $Y=[f(x-$ $\left.\left.c t_{1}\right), \ldots, f\left(x-c t_{n}\right)\right], t_{1}=0, t_{n}=L, n=185$, and computing its singular value decomposition leads to a slow decay of singular values, see Fig. 2a. However, if the transport is compensated by a time-dependent shift $\mathcal{T}^{-\Delta^{t}}(f(t, x)):=f(t, x+c t)$ and $\Delta^{t}:=c t$, which transforms the traveling pulse into a stationary frame, we need only a few modes to decompose the data up to machine precision, see Fig. 2b.

Generalizing the concept introduced in the previous paragraph and noting that there may be multiple transports in the studied system, the low-dimensional approximation $y^{\ell}$ of the full order 

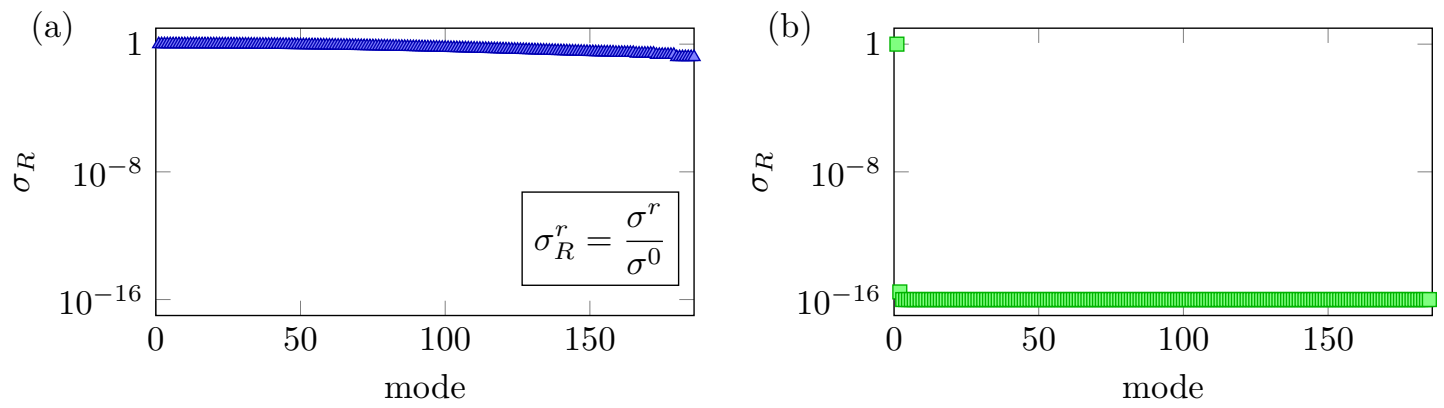

Figure 2: Right traveling wave for a Gaussian pulse, scaled singular values $\left(\sigma_{R}\right)$ decay (a) for a direct POD application, (b) after transforming the data from a co-moving frame into a stationary one.

model unknown $y$ is sought in the form

$$
y^{\ell}(x, t)=\sum_{k=1}^{N_{\mathrm{f}}} T^{\Delta_{k}^{t}}\left(\sum_{r=1}^{\ell_{k}} \psi_{r}^{k} \otimes \eta_{r}^{k}\right)=\sum_{k=1}^{N_{\mathrm{f}}} T^{\Delta_{k}^{t}}\left(\Psi_{k}^{\ell_{k}} H_{k}^{\ell_{k}}\right),
$$

where $N_{\mathrm{f}}$ is the number of frames of reference, i.e. the number of transports in the studied system. However, using (8) in model order reduction has a few specifics. First, the transport operators $T^{\Delta_{k}^{t}}, k=1, \ldots, N_{\mathrm{f}}$, have to be known a-priori, which requires a level of physical insight into the studied system behavior. Next, $T^{\Delta_{k}^{t}}, k=1, \ldots, N_{\mathrm{f}}$ have to be, up to numerical errors, invertible and continuous with respect to time and, for time-parameter dependent systems, with respect to other system parameters. Finally, decomposing the source data $Y$ into $N_{\mathrm{f}}$ frames and embedding each decomposition $\Psi_{k}^{\ell_{k}} H_{k}^{\ell_{k}}$ in a unique operator disallows using any $\Psi_{k}^{\ell_{k}}, k=1, \ldots, N_{\mathrm{f}}$, for projection of the system (4) from the full order space $V$ into a single low-dimensional subspace $V^{\ell}$.

Note that the shifted proper orthogonal decomposition was not yet mentioned in the present section. The reason being that the general idea of applying a transport operator to the source data in order to improve their decomposition properties is not unique to it, see e.g. [7,8]. The uniqueness and specificity of sPOD lies in splitting the data in $Y$ into $k$ individual frames of reference, where each frame contains a transport describable by $T^{\Delta_{k}^{t}}$. The data sorting is implemented as an optimization problem that performs $N_{\mathrm{f}}$ SVD decompositions in each iteration. In other words, each optimization problem iteration is approximately $N_{\mathrm{f}}$ times more computationally demanding than POD. However, a detailed discussion of the sPOD algorithm is outside of this paper scope and the reader is referred to the literature [4].

PODIANN and SPODIANN frameworks The proposed approach to model reduction of transport dominated systems comprises (shifted) Proper Orthogonal Decomposition and Interpolation using Artificial Neural Networks (PODIANN if POD is used and sPODIANN for the case of shifted POD). The framework for time-dependent systems is summarized in Fig. 3. Note that the idea of coupling the proper orthogonal decomposition with machine learning approaches was already investigated, even for time-parameter dependent systems (parametrized PDEs), see e.g. [10]. However, utilizing the shifted POD variant is, up to the authors knowledge, a novelty.

The overall framework is the same for both PODIANN and sPODIANN. In the case of PODIANN, the framework is purely data-driven, i.e., the matrix $Y$ is sufficient for the ROM construction as the toposes $\Psi^{\ell}$ and chronoses $H^{\ell}$ in the decomposition (1) are obtained from SVD of $Y$ and a time-continuous ROM is obtained by training an ANN on $H^{\ell}$ to provide a chronos estimate $\tilde{\eta}^{\ell}$ for any $t \in[0, T]$. Note that for the purely time-dependent case and PODIANN, using ANNs to estimate $\tilde{\eta}^{\ell}$ is rather ineffective as they can be replaced by standard interpolation methods. However, interpolation may not be sufficient when moving towards parametrized systems.

The sPODIANN framework, in which the shifted POD is used, needs to account for the transport operators. First, the method is no longer purely data driven because a-priori knowledge of at least $\left\{T^{\Delta_{k}^{t}}\right\}_{k=1}^{N_{\mathrm{f}}}$, the discrete version of the transport operators, is required. Furthermore, either directly the continuous $\left\{\mathcal{T}^{\Delta_{k}^{t}}\right\}_{k=1}^{N_{\mathrm{f}}}$ or their estimates are required in the framework online stage. Note that if only $\left\{T^{\Delta_{k}^{t}}\right\}_{k=1}^{N_{\mathrm{f}}}$ are known, ANNs may be once more applied for the construction of $\left\{\tilde{\mathcal{T}}^{\Delta_{k}^{t}}\right\}_{k=1}^{N_{\mathrm{f}}}$. For a time-dependent system and a general transport operator in three dimensions, 


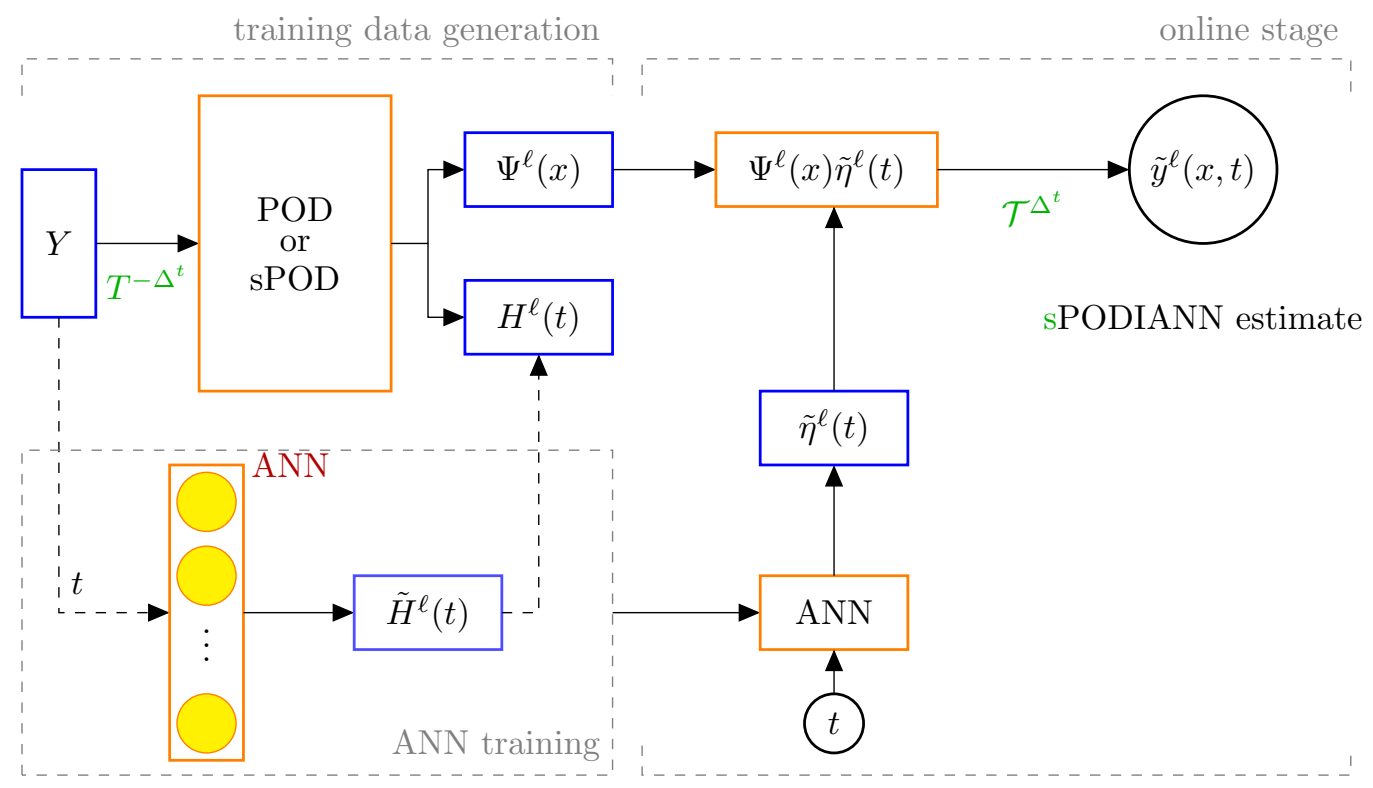

Figure 3: PODIANN and SPODIANN framework architecture. The modifications required to replace POD by sPOD for time-dependent systems are given in green.

the estimator needs to provide a mapping $I \rightarrow \mathbb{R}^{6}$, i.e. for each time $t \in(0, T]$ a positional and rotational vectors $\boldsymbol{x}, \boldsymbol{\theta} \in \mathbb{R}^{3}$ need to be estimated.

The most limiting factor of the sPODIANN framework practical applicability lies in the offline stage computational costs, i.e. in the training data generation and ANN training as depicted in Fig. 3. In particular, the training data generation requires sPOD decomposition of the matrix $Y$, which by itself comprises a high-dimensional optimization problem requiring repeated SVD of $Y$. Here, the shifted robust PCA [15] can alleviate costs. Furthermore, an ANN needs to be trained to estimate $\tilde{\eta}^{\ell}$ and if continuous $\left\{\mathcal{T}^{\Delta_{k}^{t}}\right\}_{k=1}^{N_{\mathrm{f}}}$ are not known, another ANN is required to provide $\left\{\tilde{\mathcal{T}}^{\Delta_{k}^{t}}\right\}_{k=1}^{N_{\mathrm{f}}}$.

\section{Numerical examples}

The PODIANN and sPODIANN frameworks capabilities are illustrated on three numerical examples. First, we focus on the canonical case of a laminar flow around a cylindrical obstacle, the von Kármán vortex street, and compare the PODIANN framework results with a standard PODDEIM approach published in [1]. Next, two transport-dominated systems are used to illustrate the sPODIANN behavior for cases with multiple co-moving frames. In particular we have chosen a case with two discs moving with different prescribed velocities; and a case with two impellers rotating inside a square cavity.

von Kármán vortex street The von Kármán vortex street displays behaviour convenient for POD-based reduced order modeling. Specifically, at low Reynolds numbers, the shedding of the vortices is periodic. In the studied case of $\mathrm{Re}=100$, only a few POD modes is needed to construct a ROM of sufficient accuracy.

The full order model used to generate data corresponded to a finite volume simulation performed on a mesh comprising 17900 cells. Altogether 751 snapshots of flow variables were saved and used to prepare a POD-DEIM and PODIANN ROMs of dimensione $\ell=6$. In Fig. 4 , we show the test case results for the velocity field. The decay of scaled singular values for the matrix of snapshots is depicted in Fig. 4a. In Fig. 4b, we compare the time evolution of a space-mean relative error $\varepsilon_{R}$ (see the definition in Fig. 4a, where $\bar{y}=\max _{\forall t}\{\max \{|y(t)|\}\}$ and $\left.\underline{y}=\min _{\forall t}\{\min \{|y(t)|\}\}\right)$, between the FOM and ROM. Note that $\varepsilon_{R}$ achieved with PODIANN, i.e. using POD with temporal interpolation via ANNs, is of the same order of accuracy as achieved with POD-DEIM approach used in [1]. Furthermore, the qualitative results of FOM, PODIANN, and POD-DEIM are indistinguishable by naked eye, see Fig. 4c-e. 
(a)
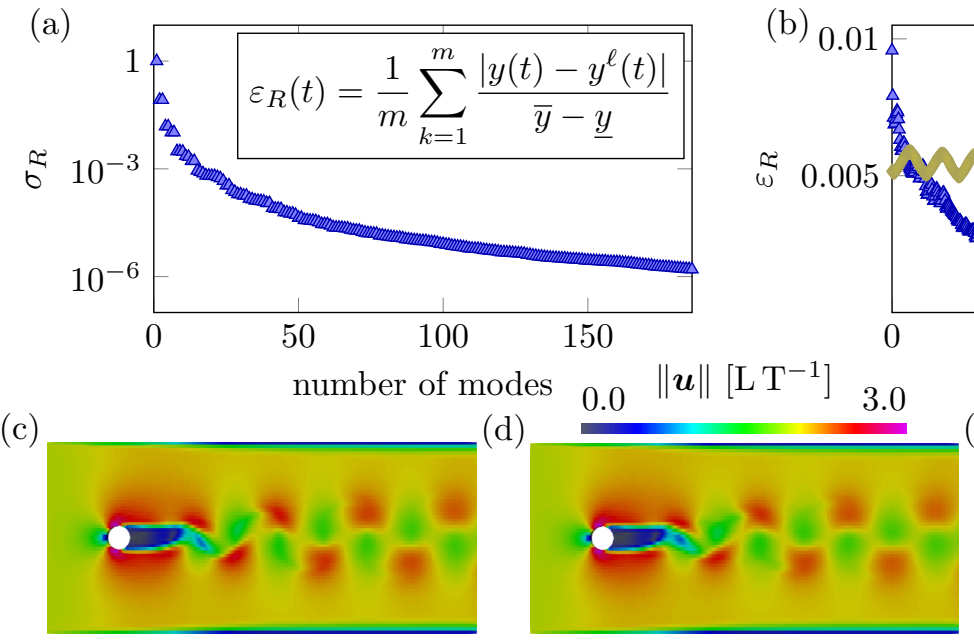

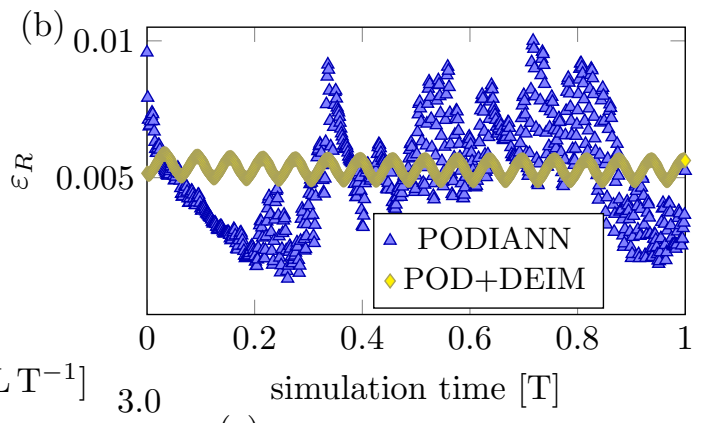

(e)

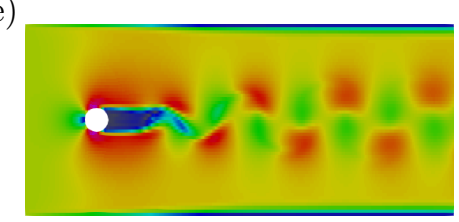

Figure 4: Von Kármán vortex street, (a) singular value decay, (b) comparison of time evolution of the global relative error between ROM and FOM for POD-DEIM and PODIANN, (c) - (e) qualitative view of the velocity field at $t=20$ for FOM, PODIANN and POD-DEIM, respectively.

Moving discs and POD approach failure The first transport-dominated system corresponds to two discs moving inside a periodic rectangular domain with two different velocities $c_{1}=0.2 \mathrm{~L} \mathrm{~T}^{-1}$ and $c_{2}=0.35 \mathrm{~L} \mathrm{~T}^{-1}$, respectively. The domain $\Omega$ of length $L=1 \mathrm{~L}$ and width $W=0.2 \mathrm{~L}$ was discretized into $30000 \mathrm{FV}$ cells. The full order model was computed with the CFD-DEM solver described in [12]. The solver tracks the position of moving solids via a scalar field $\lambda: \Omega \rightarrow[0,1]$, where $\lambda=1$ inside the solid phase, $\lambda=0$ in fluid and $\lambda \in(0,1)$ on the fluid-solid interface. Note that because of the solver architecture, (i) no evolution equations are available for the $\lambda$ field, and (ii) the solver is capable of constructing the discrete transport operators for each solid body.

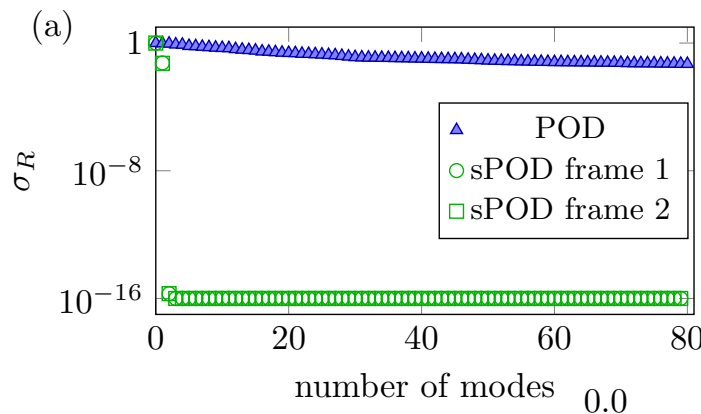

(c)

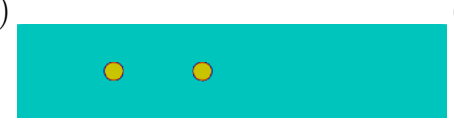

(d)

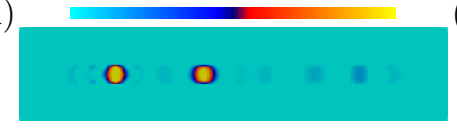

(b)

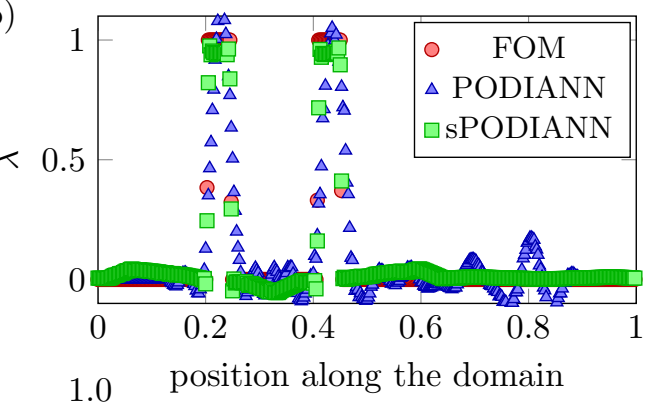

(e)

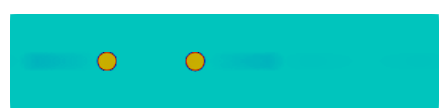

Figure 5: Two moving discs, $\lambda$ (a) singular value decay for POD and first frame of sPOD, (b) slice of the $\lambda$ field at $W / 2$ in FOM, PODIANN and sPODIANN reconstruction, (c) - (e) qualitative view of the $\lambda$ field at $t=0.42$ for FOM, PODIANN and sPODIANN, respectively.

In Fig. 5, we show similar results as in Fig. 4, this time for the $\lambda$ field from the CFD-DEM solver. Note the slow singular value decay obtained via POD in Fig. 5a and even with 20 spatial modes the POD-based ROM results are unacceptable, see Fig. 5b and d. In contrast, sPOD is capable of sorting the data in frames corresponding to each moving discs. Consequently, a single topos is required to describe each disc, sPOD returns only two non-zero singular values, and using these two toposes is sufficient to prepare a ROM of sufficient accuracy.

The velocity field $(\boldsymbol{u})$, which is generated by the discs movements, behaves similarly to the $\lambda$ field, cf. Fig. 5 and Fig. 6 . This time, the POD ROM is based on 12 spatial modes, while the sPOD one on two modes for each of the two frames (four modes in total). Once more, the sPODIANN framework results are qualitatively better than the PODIANN ones, cf. Fig. 6e and d. 

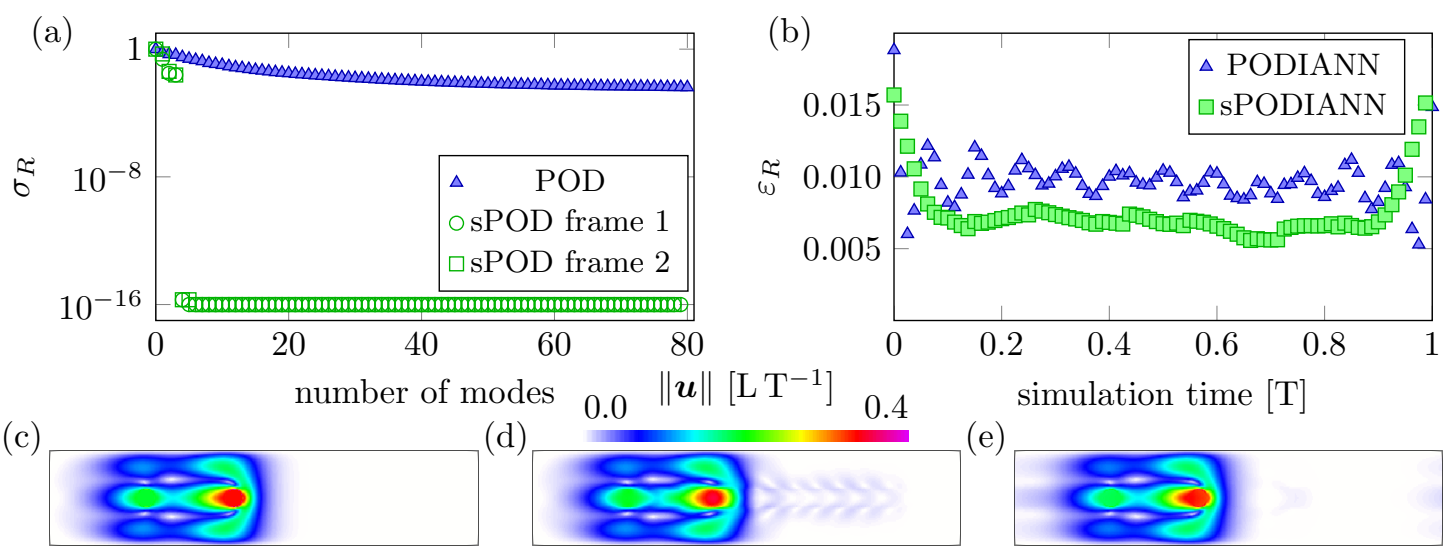

Figure 6: Two moving discs, $\boldsymbol{u}$, (a) singular value decay for POD and first frame of sPOD, (b) comparison of time evolution of the global relative error between ROM and FOM, (c) - (e) qualitative view of the velocity field at $t=0.42$ for FOM, POD and SPOD, respectively.

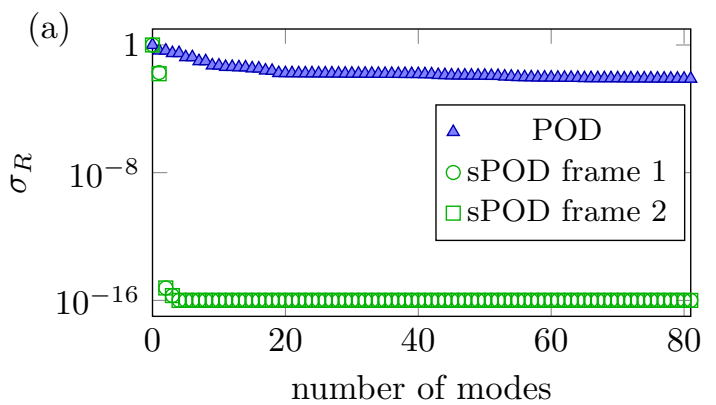

(c)

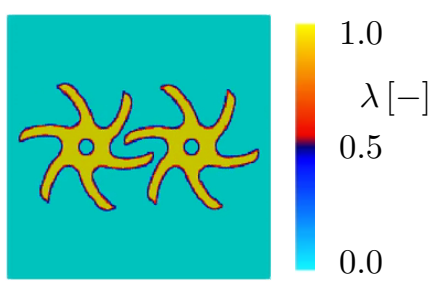

(b)

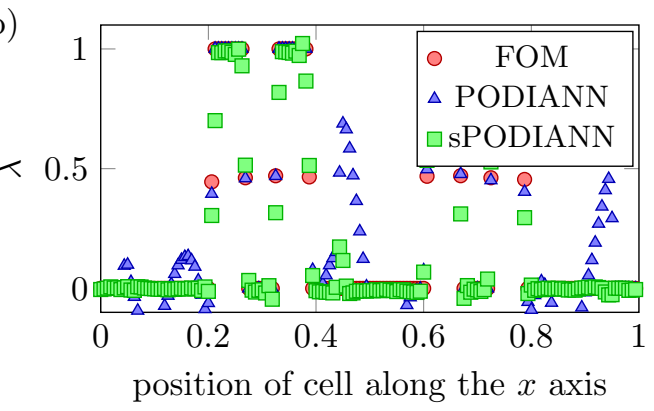

(e)

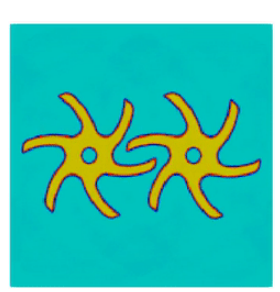

Figure 7: Two rotating impellers, (a) singular value decay for POD and first frame of sPOD, (b) slice of the $\lambda$ field at $W / 2$ in FOM, PODIANN and sPODIANN reconstruction (c) - (e) qualitative view of the $\lambda$ field at $t=1.005$ for FOM, POD and sPOD, respectively.

Rotating impellers The last studied case is based on two rotating impellers in a square cavity of $W=L=1 \mathrm{~L}$. The two impellers rotate with angular velocities of $\pm 2 \pi \operatorname{rad~T}^{-1}$. Consequently, the transformation operators $\mathcal{T}^{\Delta_{k}^{t}}$ describe the impellers rotation and are defined by $\Delta_{k}^{t}:=(-1)^{k}$. $2 \pi \boldsymbol{r}_{k} t, k=1,2$, where $\boldsymbol{r}_{k}$ is a vector field defining radial coordinates of the points in $\Omega$ with respect to the $k$-th impeller centroid. In Fig. 7, we show results for the $\lambda$ field analysis. Once more, nonphysical oscillations may be identified in POD-based ROM results, see Fig. 7b and d. These oscillations are significantly less pronounced in the sPOD-based approach, Fig. 7b and e.

\section{Conclusion}

In this contribution, we have outlined a framework for combination of the shifted proper orthogonal decomposition with interpolation via artificial neural networks (sPODIANN). The shifted POD significantly improves the dimensionality reduction efficiency for systems with dominant transport and ANNs allow construction of a reduced order model for cases, in which only the discrete analogue of the transport operators and no other information on the full order model (FOM) is known. The proposed framework behavior was illustrated on three cases, the von Kármán vortex street, where no shifts were needed and a simple comparison between a standard POD-DEIM and ANN- 
based approach was possible and two different transport dominated systems where we compared the sPOD-based framework with its POD-based variant. The studied transport dominated systems comprised two discs and two impellers moving with prescribed translational and rotational velocities, respectively. The sPOD-based framework outperformed the POD-based one for both systems. In the following work, the sPODIANN framework will be extended for parametrized systems. A special focus will be made on FOMs generating discrete analogues of sPOD transport operators.

\section{Acknowledgment}

The work was financially supported by the Czech Science Foundation (GA19-22173S), by the institutional support RVO:61388998, and by the Centre of Excellence for Nonlinear Dynamic Behaviour of Advanced Materials in Engineering CZ.02.1.01/0.0/0.0/15_003/0000493 (Excellent Research Teams) in the framework of Operational Programme Research, Development and Education. Philipp Krah gratefully acknowledges the support of the Deutsche Forschungsgemeinschaft (DFG) as part of GRK2433 DAEDALUS.

\section{References}

[1] Isoz, M.: POD-DEIM based model order reduction for speed-up of flow parametric studies. Ocean Eng. vol. 186: (2019). pp. 106083-1-106083-17. doi: 10.1016/j.oceaneng.2019.05.065.

[2] Reiss, J., Schulze, P., Sesterhenn, J. \& Merhmann, V.: The shifted proper orthogonal decomposition: a mode decomposition for multiple transport phenomena. SIAM J. Sci. Comput. vol. 40: (2018). pp. A1322-A1344. doi: 10.1137/17M1140571.

[3] Chaturantabut, S. \& Sorensen, D. C.: Nonlinear model reduction via discrete empirical interpolation. SIAM J. Sci. Comp. vol. 32: (2010). pp. 2737-2764. doi: 10.1137/090766498.

[4] Reiss, J.: Optimization-based modal decomposition for systems with multiple transports. SIAM J. Sci. Comput. vol. 43: (2021). pp. A2079-A2101.

[5] Taira, K., Hemati, M. S., Brunton, S. L., Sun, Y., Duraisamy, K., Bagheri, S., Dawson, S. T. M. \& Yeh, C.-A.: Modal analysis of fluid flows: Applications and outlook. AIAA J. vol. 58: (2020). page 2737. doi: 10.2514/1.J058462.

[6] Greif, C. \& Urban, K.: Decay of the kolmogorov n-width for wave problems. Applied Mathematics Letters. vol. 96: (2019). pp. 216-222. ISSN 0893-9659. doi: 10.1016/j.aml.2019.05.013.

[7] Rim, D., Peherstorfer, B. \& Mandli, K. T.: Manifold Approximations via Transported Subspaces: Model reduction for transport-dominated problems. arXiv e-prints. (2019). page arXiv:1912.13024.

[8] Welper, G.: Interpolation of functions with parameter dependent jumps by transformed snapshots. SIAM J. Sci. Comput. vol. 39 no. 4: (2017). pp. A1225-A1250. doi: $10.1137 / 16 \mathrm{M} 1059904$

[9] Fresca, S., Dede, L. \& Manzoni, A.: A comprehensive deep learning-based approach to reduced order modeling of nonlinear time-dependent parametrized pdes. J. Sci. Comput. vol. 87: (2021). pp. 61-1-61-36. doi: 10.1007/s10915-021-01462-7.

[10] Fresca, S. \& Manzoni, A.: POD-DL-ROM: Enhancing deep learning-based reduced order models for nonlinear parametrized pdes by proper orthogonal decomposition. Comput. Methods Appl. Mech. Engrg. vol. 388: (2022). pp. 114181-1-114181-27. doi: 10.1016/j.cma.2021.114181.

[11] Isoz, M. \& Sourek, M. POD-DEIM-based model order reduction for four-way coupled fluidsolid flows. In Simurda, D. \& Bodnar, T., editors, Proceedings of the conference Topical Problems of Fluid Mechanics: pp. 98-105. IT CAS: Prague, Czech Republic: (2020). doi: 10.14311/TPFM.2020.013.

[12] Sourek, M. \& Isoz, M. Development of CFD solver for four-way coupled particle-laden flows. In Simurda, D. \& Bodnar, T., editors, Proceedings of the conference Topical Problems of Fluid Mechanics: pp. 214-221. IT CAS: Prague, Czech Republic: (2020). doi: 10.14311/TPFM.2020.028.

[13] Volkwein, S. Proper orthogonal decomposition: theory and reduced-order modelling. Universtity of Konstanz: Konstanz, Germany: 1 edition: (2013).

[14] Pearson, K.: On lines and planes closest fit to systems of points in space. Phil. Mag. vol. 2: (1901). pp. 559-572. doi: 10.1080/14786440109462720.

[15] Krah, P., Goldack, M., Engels, T., Schneider, K. \& Reiss, J.: Data-driven reduced order modeling for flows with moving geometries using shifted pod. In 10th Vienna International Conference on Mathematical Modelling,(MATHMOD 2022): (2022). 